\title{
Application of telemedicine during the coronavirus disease epidemics: a rapid review and meta-analysis
}

\author{
Yelei Gao ${ }^{1,2,3 \#}$, Rui Liu, ${ }^{1,2,3}$, Qi Zhou ${ }^{4,5 \#}$, Xingmei Wang ${ }^{1,2,3}$, Liping Huang ${ }^{1,2,3}$, Qianling Shi ${ }^{4,5}$, \\ Zijun Wang ${ }^{5}$, Shuya $\mathrm{Lu}^{5,6,7}$, Weiguo $\mathrm{Li}^{1,2,3}$, Yanfang $\mathrm{Ma}^{5}$, Xufei Luo ${ }^{8}$, Toshio Fukuoka, ${ }^{9,10}$ Hyeong Sik Ahn ${ }^{11,12}$, \\ Myeong Soo Lee ${ }^{13,14}$, Zhengxiu Luo ${ }^{1,2,3}$, Enmei Liu ${ }^{1,2,3}$, Yaolong Chen ${ }^{5,15,16,17}$, Chang Shu ${ }^{1,2,3}$, \\ Daiyin Tian $^{1,2,3}$; on behalf of COVID-19 Evidence and Recommendations Working Group
}

\begin{abstract}
${ }^{1}$ Department of Respiratory Medicine, Children's Hospital of Chongqing Medical University, Chongqing 400014, China; ${ }^{2}$ National Clinical Research Center for Child Health and Disorders, Ministry of Education Key Laboratory of Child Development and Disorders, China International Science and Technology Cooperation Base of Child Development and Critical Disorders, Children's Hospital of Chongqing Medical University, Chongqing 400014, China; ${ }^{3}$ Chongqing Key Laboratory of Pediatrics, Chongqing 400014, China; ${ }^{4}$ The First School of Clinical Medicine, ${ }^{5}$ Evidence-based Medicine Center, School of Basic Medical Sciences, Lanzhou University, Lanzhou 730000, China; ${ }^{6}$ Department of Pediatric, Sichuan Provincial People's Hospital, University of Electronic Science and Technology of China, Chengdu 611731, China; ${ }^{7}$ Chinese Academy of Sciences Sichuan Translational Medicine Research Hospital, Chengdu 610072, China; ${ }^{8}$ School of Public Health, Lanzhou University, Lanzhou 730000, China; ${ }^{9}$ Emergency and Critical Care Center, the Department of General Medicine, Department of Research and Medical Education at Kurashiki Central Hospital, Kurashiki, Japan; ${ }^{10}$ Advisory Committee in Cochrane Japan, Tokyo, Japan; ${ }^{11}$ Department of Preventive Medicine, Korea University College of Medicine, Seoul, Korea; ${ }^{12}$ Korea Cochrane Centre, Seoul, Korea; ${ }^{13}$ Korea Institute of Oriental Medicine, Daejeon, Korea; ${ }^{14}$ University of Science and Technology, Daejeon, Korea; ${ }^{15}$ Lanzhou University, an Affiliate of the Cochrane China Network, Lanzhou 730000, China; ${ }^{16}$ Chinese GRADE Center, Lanzhou 730000, China; ${ }^{17}$ Key Laboratory of Evidence Based Medicine and Knowledge Translation of Gansu Province, Lanzhou University, Lanzhou 730000, China

Contributions: (I) Conception and design: Y Chen, E Liu; (II) Administrative support: Y Chen; (III) Provision of study materials or patients: Y Gao, R Liu, X Wang; (IV) Collection and assembly of data: R Liu, X Wang; (V) Data analysis and interpretation: Q Zhou, Y Gao, Q Shi, Z Wang; (VI) Manuscript writing: All authors; (VII) Final approval of manuscript: All authors.

\#These authors contributed equally to this work.

Correspondence to: Chang Shu; Daiyin Tian. Department of Respiratory Medicine, Children's Hospital of Chongqing Medical University, Chongqing 400014, China. Email: 400361@hospital.cqmu.edu.cn; 400942@hospital.cqmu.edu.cn.
\end{abstract}

Background: As COVID-19 has become a global pandemic, early prevention and control of the epidemic is extremely important. Telemedicine, which includes medical advice given over telephone, Internet, mobile phone applications or other similar ways, may be an efficient way to reduce transmission and pressure on medical institutions.

Methods: We searched MEDLINE, Web of Science, Embase, Cochrane, CBM, CNKI and Wanfang databases for literature on the use of telemedicine for COVID-19, SARS and MERS from their inception to March 31st, 2020. We included studies about the content of the consultation (such as symptoms, therapy and prevention, policy, public service), screening of suspected cases, the provision of advice given to those people who may have symptoms or contact history. We conducted meta-analyses on the main outcomes of the studies.

Results: A total of 2,041 articles were identified after removing duplicates. After reading the full texts, we finally included nine studies. People were most concerned about symptoms (64.2\%), epidemic situation and public problems (14.5\%), and psychological problems (10.3\%) during COVID-19 epidemic. During the SARS epidemic, the proportions of people asking for consultation for symptoms, prevention and therapy, and psychological problems were $35.0 \%, 22.0 \%$, and $23.0 \%$, respectively. Two studies demonstrated that telemedicine can be used to screen the suspected patients and give advice. One study emphasized the limited possibilities to follow up people calling hotlines and difficulties in identifying all suspect cases.

Conclusions: Telemedicine services should focus on the issues that the public is most concerned about, 
such as the symptoms, prevention and treatment of the disease, and provide reasonable advice to patients with symptoms or people with epidemic history.

Keywords: COVID-19; SARS; MERS; telemedicine; rapid review

Submitted Apr 14, 2020. Accepted for publication Apr 26, 2020.

doi: $10.21037 / \mathrm{atm}-20-3315$

View this article at: http://dx.doi.org/10.21037/atm-20-3315

\section{Introduction}

In December 2019, cases of pneumonia with unknown cause were broke out. The pathogen causing the infection was subsequently identified to be a novel coronavirus (1). On February 11th, 2020, the World Health Organization officially named the novel coronavirus pneumonia as "COVID-19" (2), and the International Committee on Taxonomy of Viruses named the novel coronavirus as "SARS-CoV-2" (3). SARS-CoV-2 can be transmitted from person to person (4-6), and the population is generally susceptible (7). Severe cases of COVID-19 are associated with acute respiratory distress syndrome, acute heart injury, shock and even death $(8,9)$. Compared with SARS (severe acute respiratory syndrome) and MERS (Middle East respiratory syndrome), COVID-19 spreads faster but has lower mortality (10-12). By March 31, 2020, the virus has spread to more than 200 countries and regions in the world, with 750,890 confirmed cases and 36,405 deaths (13), resulting in a fatality among known cases as high as $4.8 \%$. On March 11, 2020, the World Health Organization declared COVID-19 as a global pandemic.

With the rapid development of communication equipment and Internet, telemedicine has become a convenient way for the public to obtain valuable information and health consultation. Most COVID-19 patients have attended hospitals or other health facilities to be diagnosed and treated, which increases the risk of nosocomial infection (9). Remote medical treatment can reduce the unnecessary hospital visits during the outbreak and the accumulation of people in the hospital, accelerate the patients' access to professional advice in time, and alleviate anxiousness among the members of public. Discovering, diagnosing and treating patients infected with SARS-CoV-2 as early as possible support the prevention and control of the epidemic. The purpose of this rapid review is to explore the role and potential of telemedicine during the COVID-19, SARS and MERS outbreaks. We present the following article in accordance with the PRISMA reporting checklist (available at http://dx.doi.org/10.21037/atm-20-3315).

\section{Methods}

\section{Search strategy}

A comprehensive search was performed by an experienced librarian in the following electronic databases from their inception to March 31st, 2020 (14): the Cochrane Library, MEDLINE (via PubMed), EMBASE, Web of Science, CBM (China Biology Medicine disc), CNKI (China National Knowledge Infrastructure), and Wanfang databases. We made no restrictions on language or publication status. The following search was used: ("Novel coronavirus" OR "2019-novel coronavirus" OR "Novel CoV" OR "2019-nCoV" OR "2019-CoV" OR "COVID-19" OR "COVID 19" OR "SARS-CoV-2" OR "Middle East Respiratory Syndrome" OR "MERS" OR "MERS-CoV" OR "Severe Acute Respiratory Syndrome" OR "SARS" OR "SARS-CoV" OR "SARSRelated" OR "SARS-Associated") AND ("Consultants" OR "Telemedicine" OR "Internet" OR "Counseling" OR "Consultant" OR "Consult" OR "Advisory Service" OR "Advisory Services" OR "Telehealth" OR "eHealth" OR "mHealth" OR "Mobile Health" OR "Online consultation" OR "Telephone" OR "Hotline*" OR "Online Reference Service" OR "Online Reference Desk" OR "Network Information Reference" OR "Real-time Reference Service" OR "Online inquiry" OR "Mobile Application*"OR "Mobile App"” OR "Cell Phone" OR "Mobile phone*”). We also searched clinical trial registry platforms [the World Health Organization Clinical Trials Registry Platform (http://www.who.int/ictrp/en/), US National Institutes of Health Trials Register (https://clinicaltrials.gov/)], Google Scholar (https://scholar.google.nl/), preprint platforms 
[bioRxiv (https://www.biorxiv.org/), medRxiv (https://www. medrxiv.org/), SSRN (https://www.ssrn.com/index.cfm/en/)] and reference lists of the identified reviews to find unpublished and other potentially relevant studies. Finally, we contacted experts in the field to identify any relevant trials that may have been missed in our search. The details of the search strategy can be found in the Supplement I.

\section{Inclusion and exclusion criteria}

We included studies that met the following criteria: (I) the study population was people needing consultation related to COVID-19, SARS or MERS during the respective epidemics; and (II) the study focused on telemedicine (including the use of telephone hotline, telephone counseling, mobile application, Internet based consultations) and its use and the potential problems .There were no limitations of languages and study types. Duplicates, studies for which the full-text was unavailable, review articles, guidelines and expert consensus statements, and studies with specific data missing were excluded.

\section{Selection of studies}

Two reviewers (R Liu and L Huang) selected the studies independently after first eliminating duplicates. The bibliographic software EndNote was used and any discrepancies were settled by discussion, consulting a third reviewer (Q Zhou) if necessary. The reviewers screened first all titles and abstracts with the pre-defined criteria, and categorized the articles into three (eligible, not eligible, and unclear) groups. In the second step, full-texts of the potentially eligible or unclear studies were reviewed to identify the final inclusion. All reasons for exclusion of ineligible studies were recorded, and the process of study selection was documented using a PRISMA flow diagram $(15,16)$.

\section{Data extraction}

Two reviewers (R Liu and X Wang) extracted the data independently with a standard data collection form. Disagreements were resolved by consensus, and a third reviewer (Y Gao) checked the extracted data for consistency and accuracy. Data extracted included: (I) Basic information: title, first author, publication year and study design; (II) participants: baseline characteristics and sample size; and (III) results: proportions of individuals using telemedicine for different contents of consultation (e.g., symptoms, therapy and prevention, policy, public service), details of screening of suspected cases, the provision of advice given to people who had symptoms or contact history, and the limitations of telemedicine.

\section{Risk of bias assessment}

Two researchers ( $Z$ Wang and Q Shi) independently assessed the potential bias in each included study. The included studies were evaluated using appropriate assessment scales depending on the study type: for RCTs, the Cochrane Risk-of-Bias assessment tool, for cohort studies, the Newcastle-Ottawa Scale (NOS) $(17,18)$, and for cross-sectional studies, the methodology evaluation tool recommended by the Agency for Healthcare Research and Quality (AHRQ) (19).

\section{Data synthesis}

For studies on telephone hotlines, we calculated the proportions of each topic of concern among all callers, i.e. the number of individuals calling to consult on the topic under consideration divided by the total number of calls. We also collected the proportions of patients who were screened suspect case and the advice given to the caller. We did a meta-analyses of proportions, reporting the effect size (ES) with $95 \%$ confidence intervals (CI) using random-effects models (20). Two-sided $\mathrm{P}$ values $<0.05$ were considered statistically significant. Heterogeneity was defined as $\mathrm{P}<0.10$ and $\mathrm{I}^{2}>50 \%$. All analyses were performed in STATA version 14. All results are limited in $0-100 \%$.

\section{Quality of the evidence assessment}

Two reviewers ( $Z$ Wang and Q Shi) assessed the quality of evidence independently using the Grading of Recommendations Assessment, Development and Evaluation (GRADE) tool (21). We produced a "Summary of Findings" table using the GRADEpro software $(22,23)$. This table shows the overall grading of body of evidence for each prespecified outcome that will be accounted for in the meta-analysis. In this approach, the quality is downgraded according to five considerations (study limitations, consistency of effect, imprecision, indirectness, and publication bias) (24) and upgraded according to three considerations (large magnitude of effect, dose-response relation and plausible confounders or biases). Finally, the 


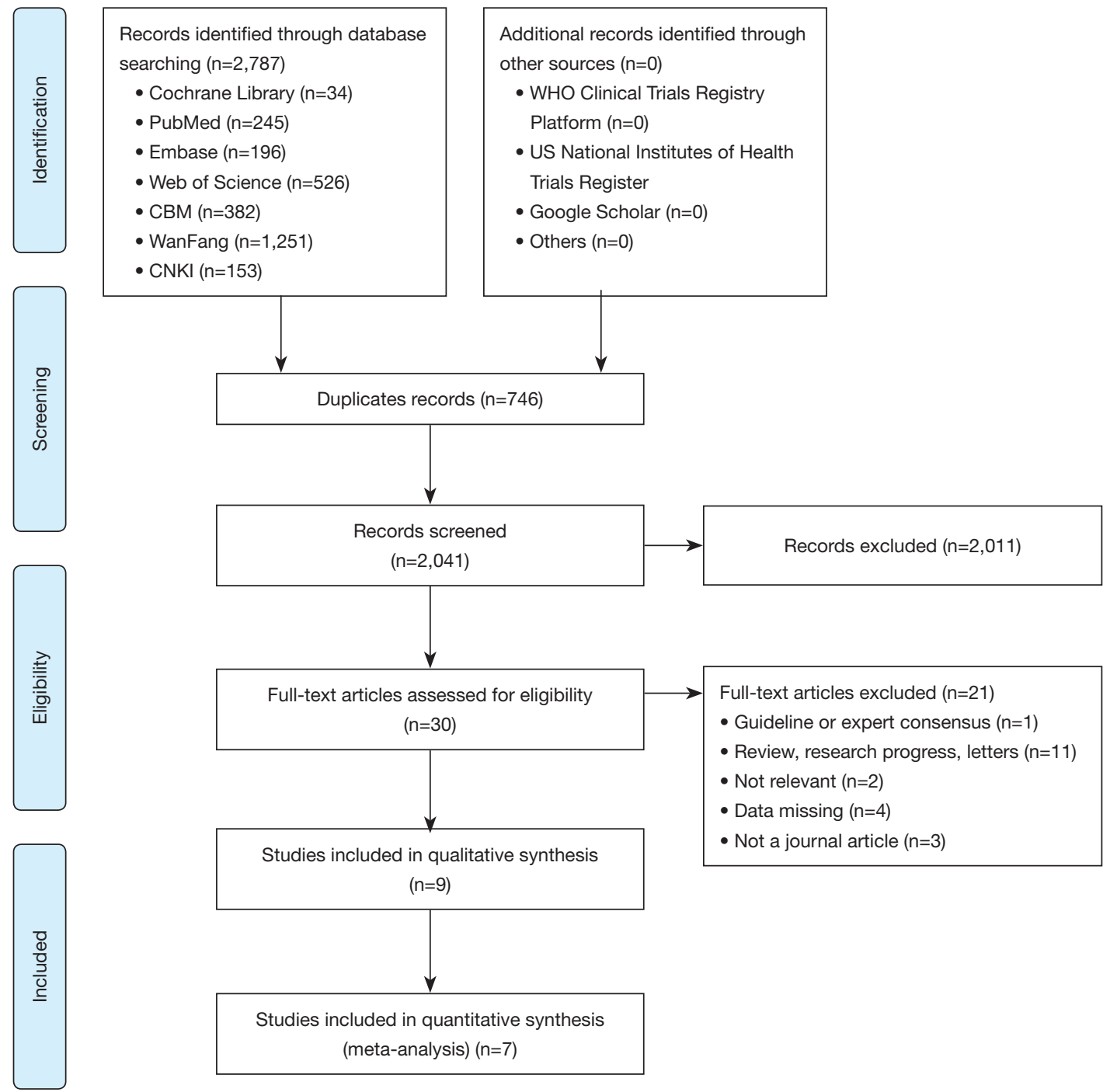

Figure 1 Flow diagram of the literature search.

quality of evidence is classified as high, moderate, low, or very low, reflecting to what extent that we are confident the effect estimates are correct.

As COVID-19 is a public health emergency of international concern and the situation is evolving rapidly, our study was not registered in order to speed up the process (25).

\section{Results}

\section{Characteristics and quality of included studies}

A total of 2,787 articles were identified in the database, of which 2,041 articles were left after deleting the duplicates.
Thirty articles were identified for further review after reviewing their titles and abstracts. Nine cross-sectional studies were finally included after reviewing the full texts (26-34). The selection process is shown in Figure 1. One of the nine retrieved articles was about COVID-19, and the remaining eight articles were about SARS. Eight articles assessed hotline consultation. The contents of the consultation included for instance symptoms, prevention and therapy, psychological problems, and related policies. Characteristics of the included studies were shown in Table 1. The quality of included studies was very poor: all studies scored less than 8 out of 11 in the evaluation by the AHRQ tool (Table 2). 


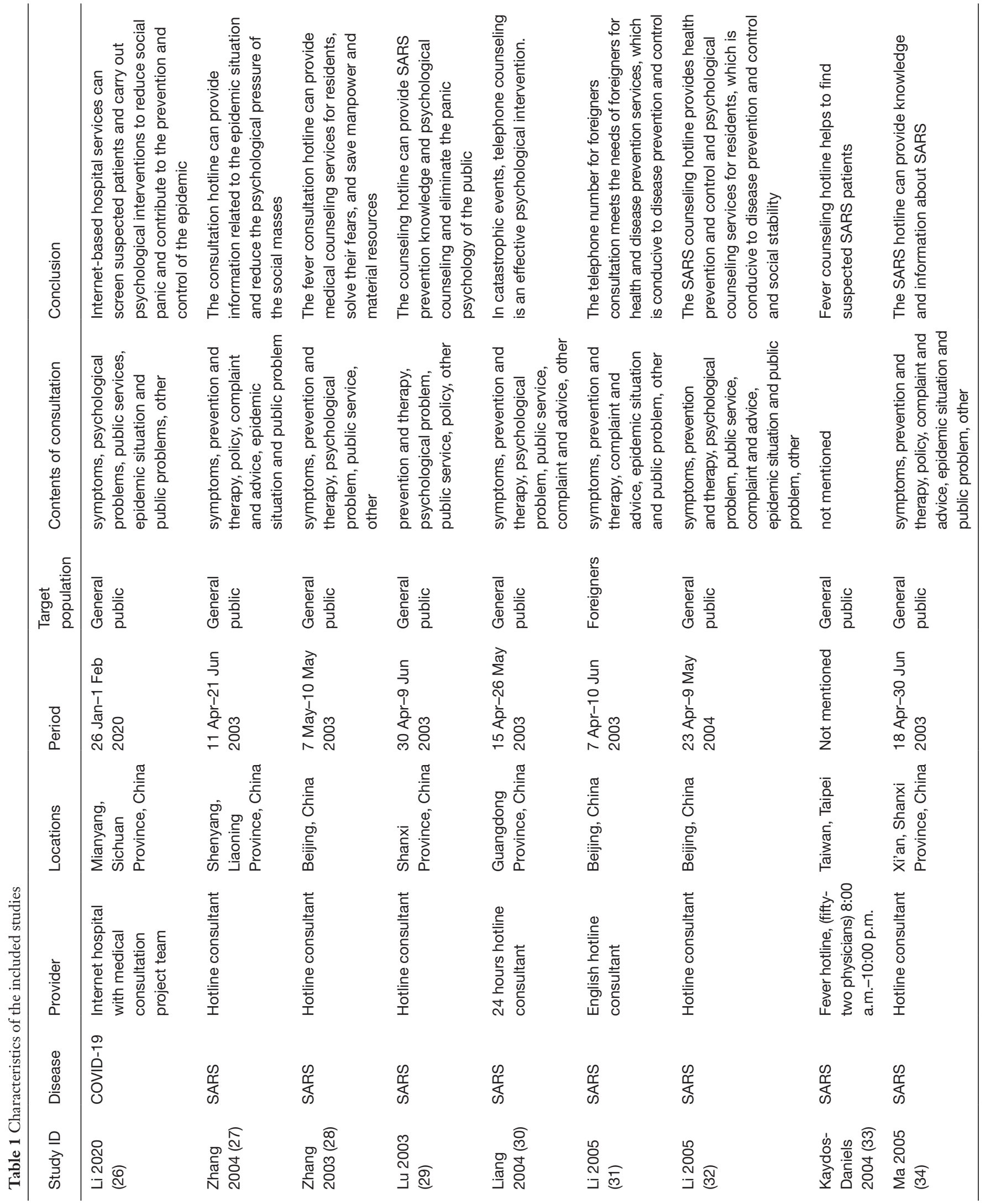


Table 2 Risk of bias in the included studies

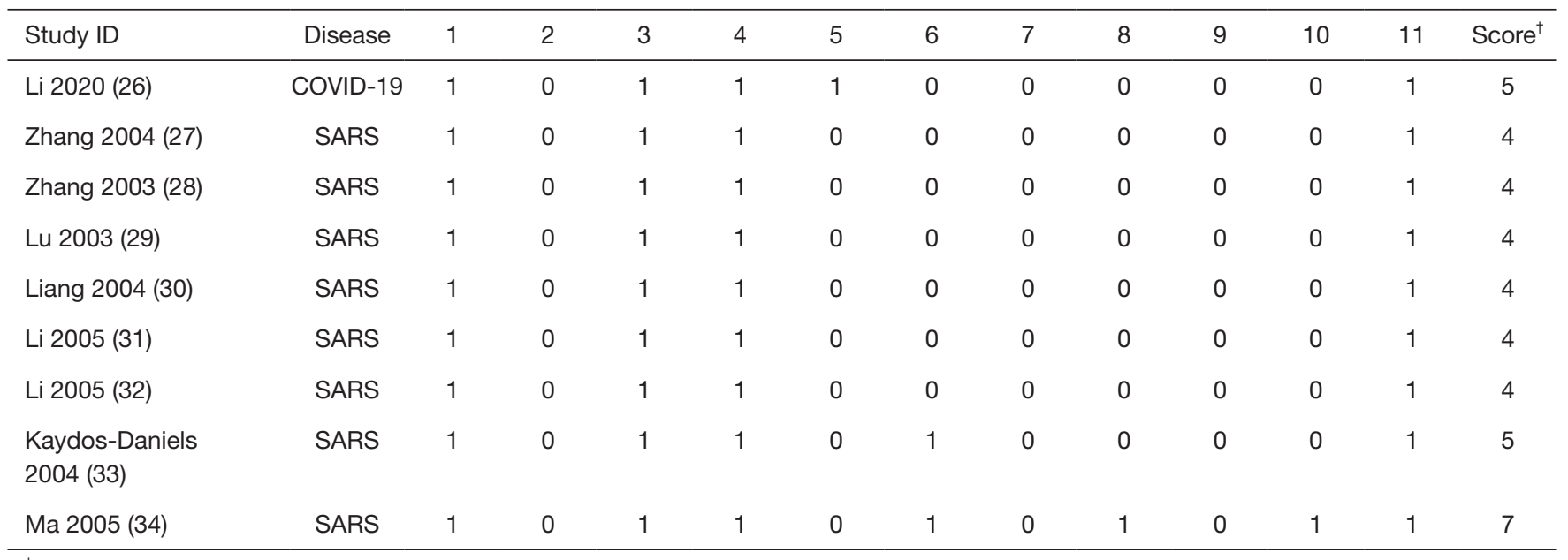

${ }^{\dagger}$, according to the methodology evaluation tool recommended by the Agency for Healthcare Research and Quality. The maximum score is 1; the higher the score, the lower the risk of bias. The numbers 1 to 11 refer to the items of the tool: 1 . Defining the source of information (survey, record review); 2. Listing the inclusion and exclusion criteria for exposed and unexposed subjects or referring to previous publications; 3. Indicate time period used for identifying patients; 4. Indicating whether the subjects were recruited consecutively (if not population-based); 5 . Indicating if evaluators of subjective components of the study were masked from the participants; 6 . Description of any assessments undertaken for quality assurance purposes (e.g., test/retest of primary outcome measurements); 7 . Explaining any exclusions of patients from the analysis; 8. Description how confounding was assessed and/or controlled; 9. If applicable, explaining how missing data were handled in the analysis; 10. Summarizing patient response rates and completeness of data collection; 11. Clarification of the expected follow-up (if any), and the percentage of patients with incomplete data or follow-up.

\section{Contents of the consultation content}

Table 3 summarizes the quantitative findings of each study. Seven studies of SARS were conducted meta-analysis in different consultation contents (Figure 2).

\section{Symptoms}

A total of seven studies (one on COVID-19, six on SARS) reported the proportion of patients who called the hotline for counseling related to symptoms (such as fever, cough, and gastrointestinal symptoms). The proportion was $64.2 \%$ in the study conducted during the COVID-19 epidemic, and $36.0 \%, 95 \%$ CI: 0.16 to $0.56\left(\mathrm{I}^{2}=99.9 \%\right)$ in the studies during the SARS epidemic (Figure 2).

\section{Prevention and therapy}

Seven studies reported the proportions of patients counseled for prevention and treatment (including disinfection, isolation, ventilation, drug treatment, and vaccination). All seven studies were about SARS. The pooled proportion was 23.0\%, 95\% CI: 0.13 to 0.33 ( $\mathrm{I}^{2}=99.9 \%$ ) (Figure 2).

\section{Psychological problems}

Five studies (one on COVID-19, four on SARS) reported the proportion of patients receiving psychological counseling (counseling content includes anxiety, worry and fear of infection related to COVID-19 or SARS). The proportion of psychological consultation is $10.3 \%$ in the COVID-19 study and $22.0 \%$ in the SARS studies, $95 \% \mathrm{CI}$ : 0.01 to $0.43\left(\mathrm{I}^{2}=99.8 \%\right)$ (Figure 2$)$.

\section{Services and advice}

Five studies (including one on COVID-19) reported the proportion of patients receiving consultation about services and advice (such as which hospital to visit if suspecting coronavirus infection, whether it is advisable to travel or organize meetings or events. The proportion was $5.6 \%$ during in the COVID-19 study and was $16.0 \%, 95 \%$ CI: 0.05 to $0.28\left(\mathrm{I}^{2}=99.3 \%\right)$ in the SARS studies (Figure 2).

\section{Policy}

Two studies, both on SARS, reported the proportion of patients receiving policy consultations (meaning e.g. local SARS prevention and control policies, SARS medical insurance reimbursement policies, or hospitalization expenses). The respective pooled proportion was $18.0 \%$, 95\% CI: 0.08 to $0.29\left(\mathrm{I}^{2}=98.9 \%\right.$ ) (Figure 2). 


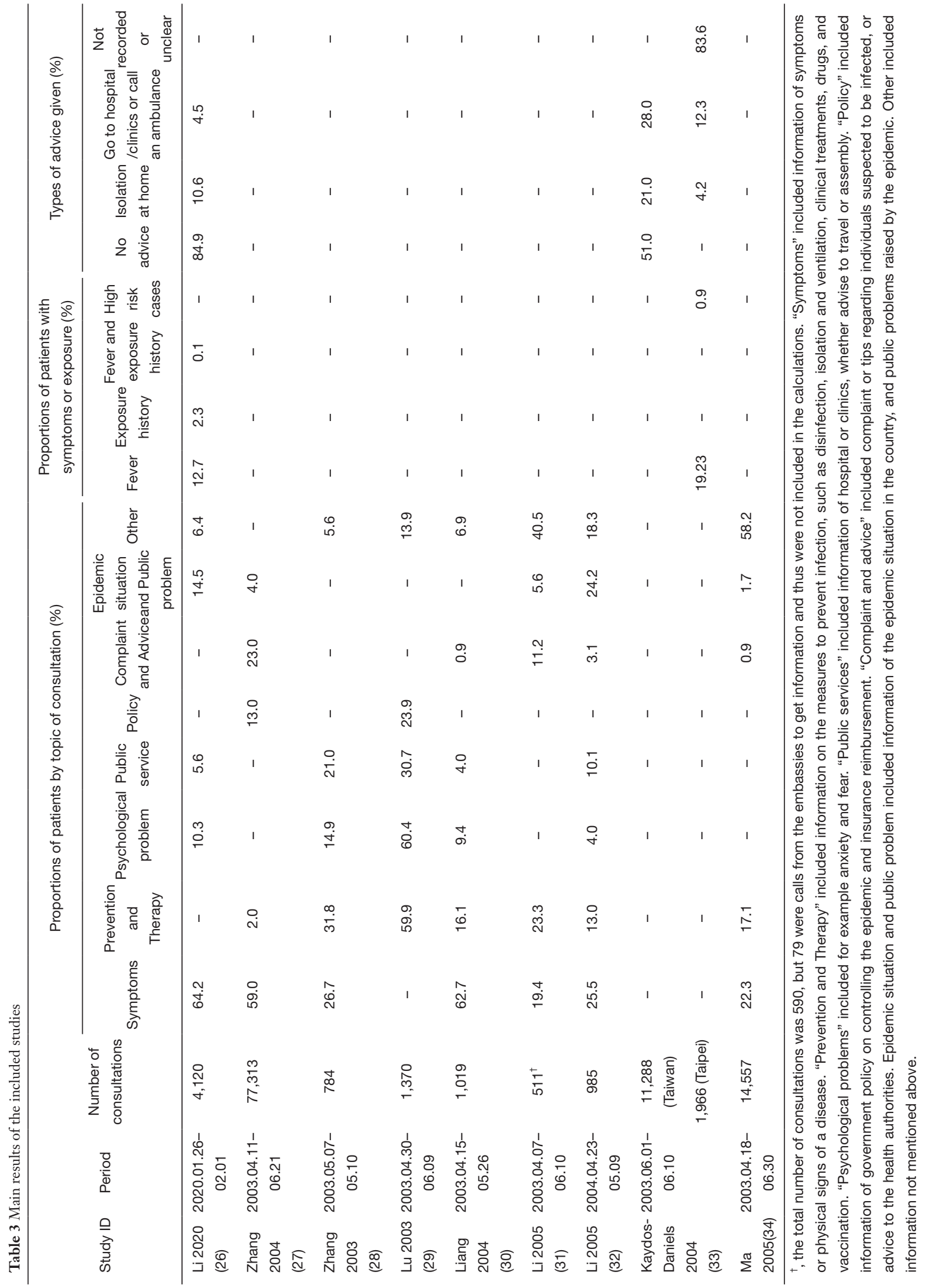




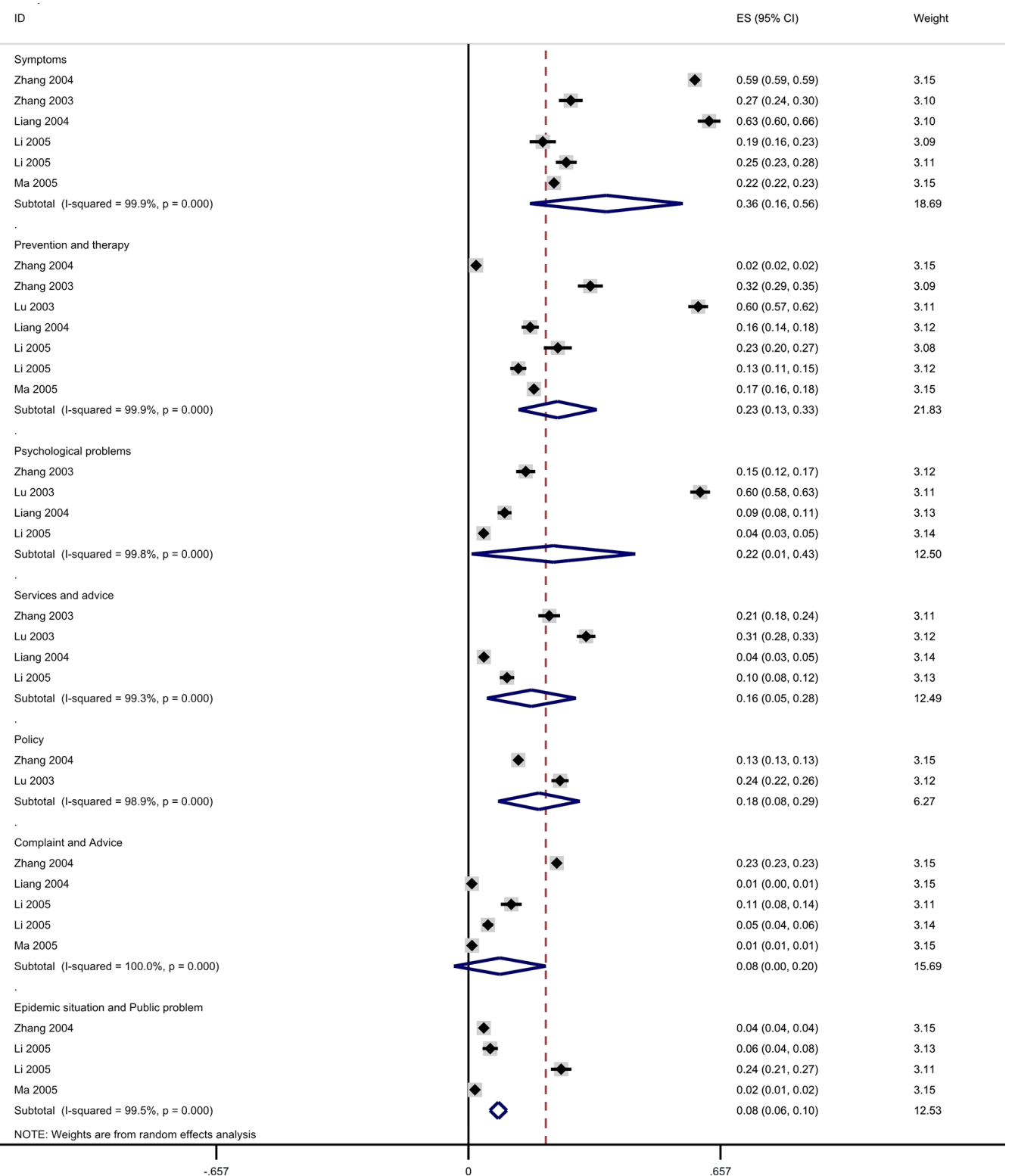

Figure 2 Forest plot on proportions of patients receiving consultation for different types of content of SARS.

\section{Complaint and advice}

A total of five studies focused on the proportion of reported complaints and advice (consultation content includes for example reporting suspected patients, or people with close contact with confirm case, and complaints of policy implementation). All the five studies were related to SARS. The results of meta-analysis of the random effect model show that the proportion of reporting and complaint consultation is $8.0 \%, 95 \%$ CI: 0.00 to $0.20\left(\mathrm{I}^{2}=100.0 \%\right)$ (Figure 2 ).

\section{Epidemic situation and public problem}

Five studies (including one on COVID-19) assessed the epidemic situation and the proportion of consultation on public issues (including disease knowledge, epidemic situation and public issues of COVID-19/SARS). The respective proportion was $14.5 \%$ in the COVID-19 study, and $8.0 \%$ in the studies conducted during the SARS epidemic (95\% CI: 0.06 to $\left.0.10 ; \mathrm{I}^{2}=99.5 \%\right)$ (Figure 2). 


\section{Using telemedicine to initially screen patients for suspected coronavirus infection}

Two studies assessed the screening of COVID-19 or SARS. The study on COVID-19 showed that among 4,120 people who needed consultation, 524 had fever, 93 had a history of exposure though contacts; five patients had both fever and contact exposure. The other study on SARS from Taipei showed that of the 1966 patients, 378 had fever, and 18 were considered to be at high risk of having SARS.

\section{Advice provision by telemedicine}

Two studies assessed the provision of advice for patients with COVID-19 or SARS given by medical experts. The study on COVID-19 showed that 437 (10.6\%) of the 4,120 patients were advised to stay at home for medical observation and 185 (4.5\%) were advised to go to a hospital or clinic, or call an ambulance. The other study on SARS showed that of the 11,288 patients, $21.0 \%$ were advised to isolate at home for observation, and $28.0 \%$ were advised to go to the clinic, hospital or call an ambulance.

\section{Limitations of telemedicine}

One studies of SARS pointed out that because people were not followed up for outcomes and hotline data were not collected systematically, it is impossible to determine how many of the patients who were suspected to be at risk of having SARS based on the telemedicine consultation were subsequently tested.

\section{Quality of evidence}

The results of GRADE on main outcomes showed that the quality of evidence on consultation contents were low or very low. The details can be found in Table S1.

\section{Discussion}

Our study showed that among the people who need consultation, COVID-19 patients were most concerned about symptoms, the epidemic situation and public problems related to the disease, whereas SARS patients were most concerned about symptoms, prevention and treatment, and psychological problems. Internet based health services and telephone hotlines were also shown to be able to initially identify some suspected patients and provide medical advice. Internet based health services and telephone hotlines can help to identify people with fever, exposure history or high-risk and make suggestions depending on their condition. The consultants providing telemedicine services were usually medical experts $(26,33)$.

According to the current knowledge, SARS-CoV-2 is mainly transmitted by droplets and direct contact, and family clusters and nosocomial infection are also common $(9,35,36)$. It is important to reduce transmission as much as possible. During infectious diseases epidemics, the public can efficiently get advice and assessment for the disease through telephone hotlines, other telemedicine services and online hospital services. This way the risk of exposing of uninfected people can also be mitigated.

We found that people were not only concerned about the disease itself, but they also needed other kinds of help during the epidemics. People who are isolated because of an infectious disease may develop negative psychological reactions, such as post-traumatic stress symptoms, confusion, anger, and fear of infection (37). Telephone hotlines can be used as a tool for psychological interventions after the outbreak of the epidemic (30), so that people can obtain the necessary knowledge and a platform to relieve their anxiety and fear.

During the outbreak of COVID-19, hospitals have implemented outpatient pharmaceutical care using an Internet based medical care model. This model helps to provide medication for patients with chronic diseases without them needing to leave home, and ensure the sustainability of medication for patients with chronic diseases and reduce the risk of potential cross-infection. Internet-based medical care also has been shown to save nearly 3.5 hours of time for prescribing medicine during follow-up, 1.9 hours of time spent in hospital, and about 55.6 CNY of travel and meal expenses for per patient (38) on $1^{\text {st }}$ to $7^{\text {th }}$ February, in West China Hospital of Sichuan University during the epidemic.

Telephone and Internet services are being widely used for influenza counselling and surveillance (39-41). According to a prospective study on influenza, a self-triage Internet based decision-making tool could help parents and adult caregivers to determine when children with influenzalike illnesses need to go to the emergency department. Fourteen of the 15 patients who eventually needed to go to the emergency department were classified as high risk by this tool, resulting in a sensitivity of $93.3 \%$ (95\% CI, $68.1 \%$ to $99.8 \%$ ). An Internet based self-triage tool can thus be feasible (42). Self-triage may be an effective way 
to encourage appropriate practice and reduce the pressure on health system services. Another study also showed that combining telephone and Internet services can be used by primary care facilities to promote patient self-management during flu season (43), and provided patients with medical advice and oseltamivir prescriptions (39).

Although telemedicine can be applied to the early prevention and control of infectious diseases, there are still some deficiencies. For example, one study from Taiwan on SARS pointed out that the lack of systematic data collection by the telemedicine hotlines meant that it is not possible to know whether all callers who were identified as being at high risk of having SARS eventually followed their recommendations and went to the medical facility (33). Some hotlines are open 24 hours a day, meaning that it may be difficult to find operators with sufficient professional knowledge. If the operators do not have enough professional knowledge, they may provide wrong information misleading the public, or provide inappropriate medical advice, leading to a treatment delay or missed diagnoses.

From these studies, we can conclude that telemedicine on one hand provides the public with access to medical resources or information, improves the awareness of diseases, and relieves psychological stress. On the other hand, it helps to protect the privacy of the patients, prevents people from going unnecessarily to hospitals and clinics, prevents nosocomial infection, and reduces the pressure on medical institutions. Telemedicine also helps to get feedback that can be used to make decisions. The studies included in the rapid review were all from China, and there was a lack of research of coronavirus from other countries or regions. Due to economic and cultural differences around the world, the results of this study may not be applicable to other countries.

\section{Strengths and limitations}

This study is to our knowledge the first systematic and comprehensive exposition of telemedicine consultation during the COVID-19, SARS and MERS epidemics. However, our study had several limitations. We found no original studies comparing telemedicine with traditional medical services in the prevention and control of COVID-19. We also did not find any studies about telemedicine use during the MERS outbreak. In the future, more research is needed to evaluate the role of telemedicine in acute infectious diseases. It is also necessary to establish a reliable telemedicine service system as soon as possible after an outbreak of an infectious disease, to help distinguish the patients with the emerging infection from other patients.

\section{Conclusions}

Telemedicine offers the public an efficient and safe way to consult healthcare professionals about the symptoms of infectious diseases, prevention and treatment measures, public health services, psychological, and other issues that the public are mostly concerned about. This way, the public can access the medical information conveniently and quickly, and reduce the risk of exposure to the infection within hospitals or clinics. Telemedicine can help in the screening of suspected infectious disease patients, which can prevent and control early infection, reduce the spread of SARS-CoV-2, and reduce the burden of medical staff in medical institutions.

\section{Acknowledgments}

We thank Janne Estill, Institute of Global Health of University of Geneva for providing guidance and comments for our review. We thank all the authors for their wonderful collaboration.

Funding: This work was supported by grants from National Clinical Research Center for Child Health and Disorders (Children's Hospital of Chongqing Medical University, Chongqing, China) (grant number NCRCCHD-2020EP-01) to EL; Special Fund for Key Research and Development Projects in Gansu Province in 2020, to YC; The fourth batch of "Special Project of Science and Technology for Emergency Response to COVID-19" of Chongqing Science and Technology Bureau, to EL; Special funding for prevention and control of emergency of COVID-19 from Key Laboratory of Evidence Based Medicine and Knowledge Translation of Gansu Province (grant number No. GSEBMKT-2020YJ01), to YC; The Fundamental Research Funds for the Central Universities (lzujbky-2020-sp14), to YC.

\section{Footnote}

Reporting Checklist: The authors have completed the PRISMA reporting checklist. Available at http://dx.doi. org/10.21037/atm-20-3315

Conflicts of Interest: All authors have completed the ICMJE uniform disclosure form (available at http://dx.doi. 
org/10.21037/atm-20-3315). MSL serves as the unpaid editorial board member of Annals of Translational Medicine from Nov 2019 to Oct 2021. The other authors have no conflicts of interest to declare.

Ethical Statement: The authors are accountable for all aspects of the work in ensuring that questions related to the accuracy or integrity of any part of the work are appropriately investigated and resolved.

Open Access Statement: This is an Open Access article distributed in accordance with the Creative Commons Attribution-NonCommercial-NoDerivs 4.0 International License (CC BY-NC-ND 4.0), which permits the noncommercial replication and distribution of the article with the strict proviso that no changes or edits are made and the original work is properly cited (including links to both the formal publication through the relevant DOI and the license). See: https://creativecommons.org/licenses/by-nc-nd/4.0/.

\section{References}

1. Hui DS, I Azhar E, Madani TA, et al. The continuing 2019-nCoV epidemic threat of novel coronaviruses to global health - The latest 2019 novel coronavirus outbreak in Wuhan, China. Int J Infect Dis. 2020;91:264-6.

2. WHO Director-General's remarks at the media briefing on 2019-nCoV on 11 February 2020 [internet]. World Health Organization. Available online: https://www.who. int/dg/speeches/detail/who-director-general-s-remarks-atthe-media-briefing-on-2019-ncov-on-11-february-2020

3. Coronaviridae Study Group of the International Committee on Taxonomy of Viruses. The species Severe acute respiratory syndrome-related coronavirus: classifying 2019-nCoV and naming it SARS-Coronaviridae Study Group of the International Committee on Taxonomy of Viruses. The species Severe acute respiratory syndromerelated coronavirus: classifying $2019-\mathrm{nCoV}$ and naming it SARS-CoV-2. Nat Microbiol 2020;5:536-44.

4. Chan JF, Yuan S, Kok KH, et al. A familial cluster of pneumonia associated with the 2019 novel coronavirus indicating person-to-person transmission: a study of a family cluster. Lancet 2020;395:514-23.

5. Li Q, Guan XH, Wu P, et al. Early Transmission Dynamics in Wuhan, China, of Novel CoronavirusInfected Pneumonia. N Engl J Med 2020;382:1199-207.

6. Chen N, Zhou M, Dong X, et al. Epidemiological and clinical characteristics of 99 cases of 2019 novel coronavirus pneumonia in Wuhan, China: a descriptive study. Lancet 2020;395:507-13.

7. China NHCotpsRo. Diagnosis and treatment of pneumonia infected by novel coronavirus (trial version 7). J PractTradit Chin Med 2020;34:3.

8. Huang C, Wang Y, Li X, et al. Clinical features of patients infected with 2019 novel coronavirus in Wuhan, China. Lancet 2020;395:497-506.

9. Wang D, Hu B, Hu C, et al. Clinical Characteristics of 138 Hospitalized Patients With 2019 Novel CoronavirusInfected Pneumonia in Wuhan, China. JAMA. 2020;323:1061-9.

10. Peeri NC, Shrestha N, Rahman MS, et al. The SARS, MERS and novel coronavirus (COVID-19) epidemics, the newest and biggest global health threats: what lessons have we learned? Int J Epidemiol 2020. doi: 10.1093/ije/ dyaa033.

11. MERS Monthly Summary, November,2019[Internet]. World Health Organization; c2020 [cited 2020 Apr 13]. Available online: https://www.who.int/emergencies/merscov/en/

12. Summary table of SARS cases by country, 1 November 2002 - 7 August 2003[Internet]. World Health Organization; c2020 [cited 2020 Apr 13]. Available online: https://www.who.int/csr/sars/country/country2003_08_15. pdf?ua $=1$

13. Coronavirus disease 2019 (COVID-19) Situation Report - 71 Data as reported by national authorities by 10:00 CET 31 March 2020[Internet]. World Health Organization; c2020 [cited 2020 Apr 13]. Available online: https://www.who.int/docs/default-source/coronaviruse/ situation-reports/20200331-sitrep-71-covid-19. pdf?sfvrsn=4360e92b_6

14. Li L, Tian J, Tian H, et al. Network meta-analyses could be improved by searching more sources and by involving a librarian. J Clin Epidemiol 2014;67:1001-7.

15. Speakman J, Gonzalez-Martin F, Perez T. Quarantine in severe acute respiratory syndrome (SARS) and other emerging infectious diseases. J Law Med Ethics 2003;31:63-4.

16. Kim KH, Tandi TE, Choi JW, et al. Middle East respiratory syndrome coronavirus (MERS-CoV) outbreak in South Korea, 2015: epidemiology, characteristics and public health implications. J Hosp Infect 2017;95:207-13.

17. Higgins JP, Altman DG, Gøtzsche PC, et al. The Cochrane Collaboration's tool for assessing risk of bias in randomised trials. BMJ 2011;343:d5928.

18. Wells G, Shea B, O'Connell D, et al. New Castle-Ottawa 
Quality Assessment Scale-Cohort Studies [Internet]. c2020[cited 2020 Apr 13]. Available online: http://www. ohri.ca/programs/clinical_epidemiology/oxford.asp

19. Rostom A, Dubé C, Cranney A, et al. Celiac Disease. Rockville (MD): Agency for Healthcare Research and Quality (US); 2004 Sep. (Evidence Reports/Technology Assessments, No. 104.) Appendix D. Quality Assessment Forms. Available online: https://www.ncbi.nlm.nih.gov/ books/NBK35156/

20. DerSimonian R, Kacker R. Random-effects model for meta-analysis of clinical trials: an update. Contemp Clin Trials 2007;28:105-14

21. Norris SL, Meerpohl JJ, Akl EA, et al. The skills and experience of GRADE methodologists can be assessed with a simple tool. J Clin Epidemiol 2016;79:150-158.e1.

22. Guyatt G, Oxman AD, Akl EA, et al. GRADE guidelines: 1. Introduction-GRADE evidence profiles and summary of findings tables. J Clin Epidemiol 2011;64:383-94.

23. GRADE Working Group. GRADEpro Guideline Development Tool [Software]. McMaster University, 2015 (developed by Evidence Prime, Inc.). Available online: www.gradepro.org

24. Guyatt GH, Oxman AD, Vist GE, et al. GRADE: an emerging consensus on rating quality of evidence and strength of recommendations. BMJ 2008;336:924-6.

25. Ge L, Tian J, Li Y, et al. Association between prospective registration and overall reporting and methodological quality of systematic reviews: a meta-epidemiological study. J Clin Epidemiol 2018;93:45-55.

26. Li X, Li H, He M, et al. Effects of internet hospital in prevention and control of COVID-19. Chin J Mod Nurs 2020;26:E005.

27. Zhang Z, Sun B, Huang M, et al. Anlysis of the function of consultation hotline in server acute respiratory syndrome. Chin J of PHM 2004;20:280-1.

28. Zhang X, Yu Z, Li Y, et al. An analysis of the operation of a fever hotline for severe acute respiratory syndrome. Chin J Gen Pract 2003;2:196-8.

29. Lu Y, Li Y, Zhang T, et al. 1370 Atypical Counseling Hotline Cases. Chin J Health Edu 2003;19:758-9.

30. Liang YP. Psychological Analysis of 1019 Telephone Counselees during the Prevalence of Infectious Atypical Pneumonia. Chin J Health Edu 2004;20:710-2.

31. Li K, FF, Tang YW. Analysis of cases of consultation telephone of foreign affairs during atypical pneumonia in 2003. Chin J Health Edu 2005;21:476-7.

32. Li K, Sun H, Xie D, et al. Hotline counseling during epidmic of SARS in 2004. Chin J Health Edu 2005;21:305-6.
33. Kaydos-Daniels SC, Olowokure B, Chang HJ, et al. Body temperature monitoring and SARS fever hotline, Taiwan. Emerg Infect Dis 2004;10:373-6.

34. Ma X, Xie S, Yang H. Discussion of SARS hotline relevant information and changes in law. Occup Health 2005;21:241-3.

35. Bai SL, Wang J, Zhou Y, et al. Analysis of the first cluster of cases in a family of novel coronavirus pneumonia in Gansu Province. Zhonghua Yu Fang Yi Xue Za Zhi 2020;54:E005.

36. Yu P, Zhu J, Zhang Z, et al. A familial cluster of infection associated with the 2019 novel coronavirus indicating potential person-to-person transmission during the incubation period. J Infect Dis 2020. doi: 10.1093/infdis/ jiaa077.

37. Brooks SK, Webster RK, Smith LE, et al. The psychological impact of quarantine and how to reduce it: rapid review of the evidence. Lancet 2020;395:912-20.

38. Gao Y,Gao X, Jin Z, et al. Practice and discussion of outpatient pharmaceutical care based on medical network model during 2019 novel coronavirus disease (COVID-19). Chin J Hosp Pharm. 2020. Available online: http://kns. cnki.net/kcms/detail/42.1204.r.20200223.1636.006.html

39. Rothberg MB, Martinez KA. Influenza Management via Direct to Consumer Telemedicine: an Observational Study. J Gen Intern Med 2020. doi: 10.1007/s11606-020-05640-5.

40. Kellermann AL, Isakov AP, Parker R, et al. Web-based self-triage of influenza-like illness during the $2009 \mathrm{H} 1 \mathrm{~N} 1$ influenza pandemic. Ann Emerg Med 2010;56:288-294.e6.

41. Blozik E, Grandchamp C, von Overbeck J. Influenza surveillance using data from a telemedicine centre. Int J Public Health 2012;57:447-52.

42. Anhang Price R, Fagbuyi D, Harris R, et al. Feasibility of web-based self-triage by parents of children with influenza-like illness: a cautionary tale. JAMA Pediatr 2013;167:112-8.

43. Nagykaldi Z, Calmbach W, Dealleaume L, et al. Facilitating patient self-management through telephony and web technologies in seasonal influenza. Inform Prim Care 2010;18:9-16.

Cite this article as: Gao Y, Liu R, Zhou Q, Wang X, Huang L, Shi Q, Wang Z, Lu S, Li W, Ma Y, Luo X, Fukuoka T, Ahn HS, Lee MS, Luo Z, Liu E, Chen Y, Shu C, Tian D; on behalf of COVID-19 Evidence and Recommendations Working Group. Application of telemedicine during the coronavirus disease epidemics: a rapid review and meta-analysis. Ann Transl Med 2020;8(10):626. doi: 10.21037/atm-20-3315 


\section{Supplement I Search strategy}

\section{PubMed}

\#1 “COVID-19"[Supplementary Concept]

\#2 "Severe Acute Respiratory Syndrome Coronavirus

2"[Supplementary Concept]

\#3 "Middle East Respiratory Syndrome Coronavirus" [Mesh]

\#4 "Severe Acute Respiratory Syndrome"[Mesh]

\#5 "SARS Virus"[Mesh]

\#6 "COVID-19"[Title/Abstract]

\#7 "SARS-COV-2"[Title/Abstract]

\#8 "Novel coronavirus" [Title/Abstract]

\#9 "2019-novel coronavirus" [Title/Abstract]

\#10 “coronavirus disease-19" [Title/Abstract]

\#11 “coronavirus disease 2019" [Title/Abstract]

\#12 "COVID19" [Title/Abstract]

\#13 "Novel CoV" [Title/Abstract]

\#14 “2019-nCoV" [Title/Abstract]

\#15 “2019-CoV” [Title/Abstract]

\#16 "Middle East Respiratory Syndrome" [Title/

Abstract]

\#17 “MERS" [Title/Abstract]

\#18 "MERS-CoV" [Title/Abstract]

\#19 “Severe Acute Respiratory Syndrome" [Title/

Abstract]

\#20 "SARS" [Title/Abstract]

\#21 "SARS-CoV" [Title/Abstract]

\#22 "SARS-Related" [Title/Abstract]

\#23 "SARS-Associated" [Title/Abstract]

\#24 \#1-\#23/OR

\#25 “Consultant" [MeSH Terms]

\#26 "Telemedicine" [MeSH Terms]

\#27 "Internet" [MeSH Terms]

\#28 "Mobile Applications" [MeSH Terms]

\#29 “Counseling” [MeSH Terms]

\#30 "Consultant" [Title/Abstract]

\#31 “Consult" [Title/Abstract]

\#32 “Advisory Service*” [Title/Abstract]

\#33 "Telehealth" [Title/Abstract]

\#34 "eHealth" [Title/Abstract]

\#35 “mHealth" [Title/Abstract]

\#36 "Mobile Health" [Title/Abstract]

\#37 “Online consultation" [Title/Abstract]

\#38 "telephone" [Title/Abstract]

\#39 "hotline*" [Title/Abstract]

\#40 “counseling" [Title/Abstract]
\#41 "Online Reference Service" [Title/Abstract]

\#42 “Online Reference Desk" [Title/Abstract]

\#43 "Network Information Reference" [Title/Abstract]

\#44 "Real-time Reference Service" [Title/Abstract]

\#45 “Online inquiry" [Title/Abstract]

\#46 "Mobile Application*” [Title/Abstract]

\#47 "Mobile App*" [Title/Abstract]

\#48 “Cell Phone*" [Title/Abstract]

\#49 "Mobile phone*" [Title/Abstract]

\#50 "Internet" [Title/Abstract]

\#51 \#25-\#50/OR

\#52 \#24 AND \#51

\section{Embase}

\#1 'middle east respiratory syndrome coronavirus'/exp

\#2 'severe acute respiratory syndrome'/exp

\#3 'sars coronavirus'/exp

\#4 'COVID-19':ab,ti

\#5 'SARS-COV-2':ab,ti

\#6 'novel coronavirus':ab,ti

\#7 '2019-novel coronavirus':ab,ti

\#8 'coronavirus disease-19':ab,ti

\#9 'coronavirus disease 2019':ab,ti

\#10 'COVID19':ab,ti

\#11 'novel cov':ab,ti

\#12 '2019-ncov':ab,ti

\#13 '2019-cov':ab,ti

\#14 'middle east respiratory syndrome':ab,ti

\#15 ' middle east respiratory syndrome coronavirus':ab,ti

\#16 'mers':ab,ti

\#17 'mers-cov':ab,ti

\#18 'severe acute respiratory syndrome':ab,ti

\#19 'sars':ab,ti

\#20 'sars-cov':ab,ti

\#21 'sars-related':ab,ti

\#22 'sars-associated':ab,ti

\#23 \#1-\#22/ OR

\#24 'telemedicine':ab,ti

\#25 'mobile applications':ab,ti

\#26 'consultant"':ab,ti

\#27 'consult':ab,ti

\#28 'advisory service':ab,ti

\#29 'advisory services':ab,ti

\#30 'telehealth':ab,ti

\#31 'ehealth':ab,ti 


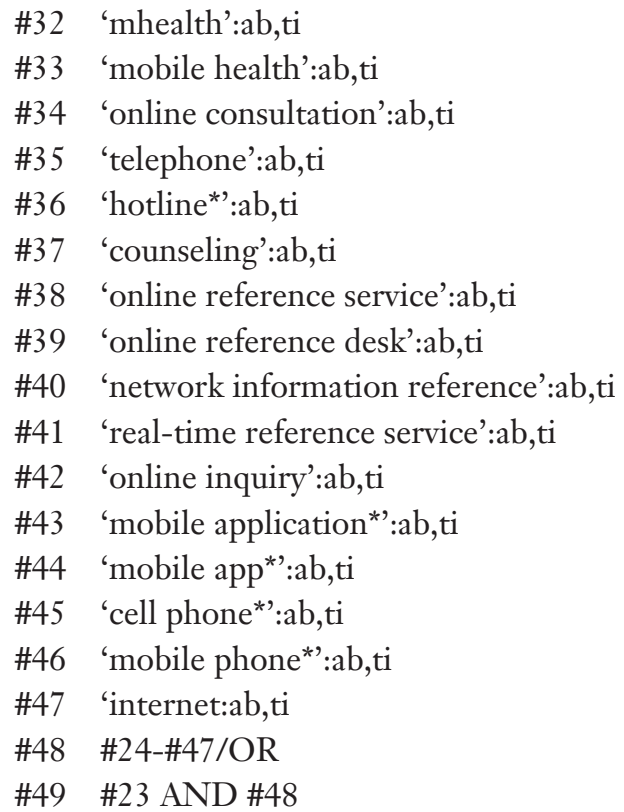

\section{Web of science}

$\begin{array}{ll}\# 1 & \text { TOPIC: "COVID-19" } \\ \# 2 & \text { TOPIC: "SARS-COV-2" } \\ \# 3 & \text { TOPIC: "Novel coronavirus" } \\ \# 4 & \text { TOPIC: "2019-novel coronavirus" } \\ \# 5 & \text { TOPIC: "coronavirus disease-19" } \\ \# 6 & \text { TOPIC: "coronavirus disease 2019" } \\ \# 7 & \text { TOPIC: "COVID 19" } \\ \# 8 & \text { TOPIC: "Novel CoV" } \\ \# 9 & \text { TOPIC: "2019-nCoV" } \\ \# 10 & \text { TOPIC: "2019-CoV" } \\ \# 11 & \text { TOPIC: "Middle East Respiratory Syndrome" } \\ \# 12 & \text { TOPIC: "MERS" } \\ \# 13 & \text { TOPIC: "MERS-CoV" } \\ \# 14 & \text { TOPIC: "Severe Acute Respiratory Syndrome" } \\ \# 15 & \text { TOPIC: "SARS" } \\ \# 16 & \text { TOPIC: "SARS-CoV" } \\ \# 17 & \text { TOPIC: "SARS-Related" } \\ \# 18 & \text { TOPIC: "SARS-Associated" } \\ \# 19 & \text { \#1-\#18/OR } \\ \# 20 & \text { TOPIC: "Online consultation" } \\ \# 21 & \text { TOPIC: "mobile Health" } \\ \# 22 & \text { TOPIC: "mHealth" } \\ \# 23 & \text { TOPIC: "eHealth" } \\ \# 24 & \text { TOPIC: "Telehealth" } \\ \# 25 & \text { TOPIC: "Advisory Service*” } \\ \# 26 & \text { TOPIC: "Consult" } \\ \# 27 & \text { TOPIC: "Counseling" } \\ \# 28 & \text { TOPIC: "Mobile Applications" }\end{array}$

\#29 TOPIC: "Internet"

\#30 TOPIC: "Telemedicine"

\#31 TOPIC: "Consultant*"

\#32 TOPIC: "Hotline*"

\#33 TOPIC: "Telephone"

\#34 TOPIC: "Counseling"

\#35 TOPIC: "Online Reference Service"

\#36 TOPIC: "Online Reference Desk"

\#37 TOPIC: "Network Information Reference"

\#38 TOPIC: "Real-time Reference Service"

\#39 TOPIC: "Online inquiry"

\#40 \#20-\#39/OR

\#41 \#19 AND \#40

\section{Cochrane library}

\#1 MeSH descriptor: [Middle East Respiratory Syndrome Coronavirus] explode all trees

\#2 MeSH descriptor: [Severe Acute Respiratory Syndrome] explode all trees

\#3 MeSH descriptor: [SARS Virus] explode all trees

\#4 “COVID-19":ti,ab,kw

\#5 "SARS-COV-2":ti,ab,kw

\#6 "Novel coronavirus":ti,ab,kw

\#7 "2019-novel coronavirus" :ti,ab,kw

\#8 "Novel CoV":ti,ab,kw

\#9 "2019-nCoV":ti,ab,kw

\#10 “2019-CoV”:ti,ab,kw

\#11 “coronavirus disease-19”:ti,ab,kw

\#12 “coronavirus disease 2019” :ti,ab,kw

\#13 “COVID19”:ti,ab,kw

\#14 "Middle East Respiratory Syndrome" :ti,ab,kw

\#15 “MERS":ti,ab,kw

\#16 "MERS-CoV":ti,ab,kw

\#17 "Severe Acute Respiratory Syndrome":ti,ab,kw

\#18 “SARS":ti,ab,kw

\#19 "SARS-CoV":ti,ab,kw

\#20 “SARS-Related":ti,ab,kw

\#21 "SARS-Associated":ti,ab,kw

\#22 \#1-\#21/ OR

\#23 “online reference desk”:ti,ab,kw

\#24 “online reference service":ti,ab,kw

\#25 “counseling”:ti,ab,kw

\#26 "hotline*":ti,ab,kw

\#27 “telephone":ti,ab,kw

\#28 “online consultation”:ti,ab,kw

\#29 “mobile health":ti,ab,kw

\#30 “mhealth":ti,ab,kw

\#31 “ehealth":ti,ab,kw 


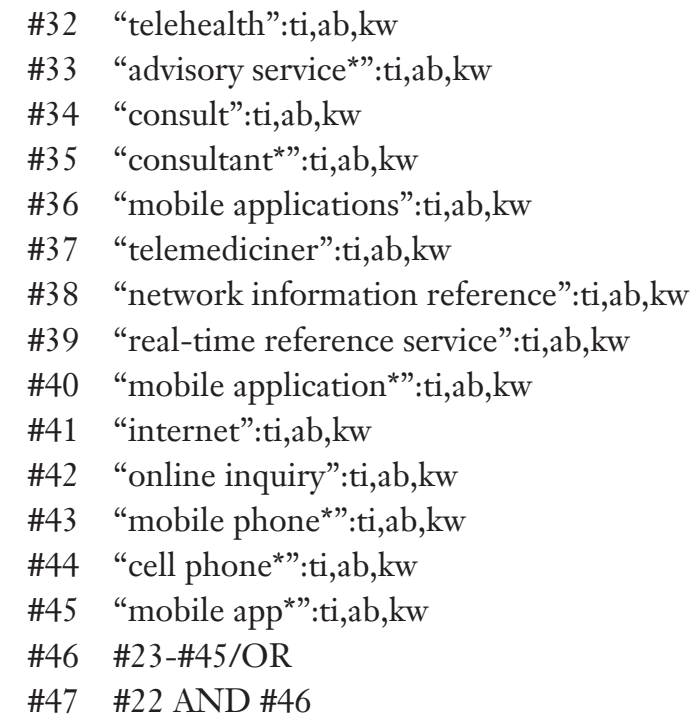

\section{CNKI}

\begin{tabular}{|c|c|}
\hline \#1 & “新型冠状病毒”[主题 ] \\
\hline \#2 & “COVID-19”[ 主题 ] \\
\hline \#3 & “COVID 19”[主题 ] \\
\hline \#4 & “2019-nCoV”[ 主题 ] \\
\hline \#5 & “2019-CoV”[ 主题 ] \\
\hline \#6 & “SARS-CoV-2”[ 主题 ] \\
\hline \#7 & “中东呼吸综合征”[主题 ] \\
\hline \#8 & “MERS”[ 主题 ] \\
\hline \#9 & “MERS-CoV”[ 主题 ] \\
\hline \#10 & “严重急性呼吸综合征” [ 主题 ] \\
\hline$\# 11$ & “SARS”[ 主题 ] \\
\hline \#12 & \#1-\#11/OR \\
\hline \#13 & “热线”[主题 ] \\
\hline \#14 & “咨询”[主题 ] \\
\hline$\# 15$ & “移动医疗”[主题 ] \\
\hline \#16 & “远程医疗”[主题 ] \\
\hline$\# 17$ & “电话”[主题 ] \\
\hline \#18 & “网络”[主题 ] \\
\hline \#19 & “因特网”[主题 ] \\
\hline \#20 & “互联网”[主题 ] \\
\hline$\# 21$ & “手机应用程序”[主题 ] \\
\hline \#22 & \#13-\#21/ OR \\
\hline \#23 & \#12 AND \#22 \\
\hline
\end{tabular}

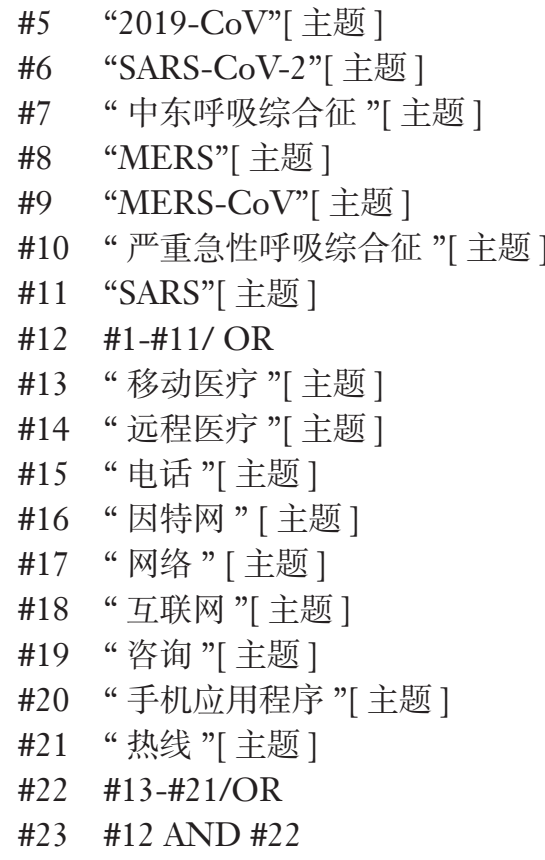

$C B M$

\#1 “新型冠状病毒”[ 常用字段 : 智能 ]

\#2“COVID-19”[ 常用字段 : 智能 ]

\#3“COVID 19”[ 常用字段 : 智能 ]

\#4 “2019-nCoV”[ 常用字段 : 智能 ]

\#5 “2019-CoV”[ 常用字段 : 智能 ]

\#6 “SARS-CoV-2”[ 常用字段 : 智能 ]

\#7 “中东呼吸综合征冠状病毒”[不加权 : 扩展 ]

\#8“中东呼吸综合征”[常用字段 : 智能 ]

\#9 “MERS”[ 常用字段 : 智能 ]

\#10 “MERS-CoV”[ 常用字段 : 智能 ]

\#11“严重急性呼吸综合征”[不加权 : 扩展 ]

\#12 “SARS 病毒”[ 不加权 : 扩展 ]

\#13 “严重急性呼吸综合征”[常用字段: 智能 ]

\#14 “SARS”[ 常用字段 : 智能 ]

\#15 \#1-\#14/OR

\#16 “移动医疗”[ 常用字段 : 智能 ]

\#17 “远程医疗”[ 常用字段 : 智能 $]$

\#18“网络”[ 常用字段 : 智能 ]

\#19 “电话”[常用字段 : 智能 ]

\#20“因特网”[常用字段: 智能 ]

\#21 “互联网”[ 常用字段: 智能 ]

\#22 “咨询”[ 常用字段: 智能 ]

\#23 “手机应用程序”[ 常用字段 : 智能 ]

\#24 “热线”[常用字段 : 智能 ]

\#25 \#16-\#24/OR

\#26 \#15 AND \#25 
Supplement II

Table S1 Summary of findings

\begin{tabular}{|c|c|c|c|c|c|c|c|c|c|}
\hline \multirow{2}{*}{ Outcomes } & \multirow{2}{*}{$\begin{array}{l}\text { No. of } \\
\text { studies }\end{array}$} & \multirow{2}{*}{$\begin{array}{l}\text { Sample } \\
\text { size }\end{array}$} & \multirow[b]{2}{*}{ Risk of bias } & \multirow[b]{2}{*}{ Inconsistency } & \multicolumn{3}{|c|}{ Certainty assessment } & \multirow{2}{*}{ Effect value $(95 \% \mathrm{Cl})$} & \multirow{2}{*}{ Certainty } \\
\hline & & & & & Indirectness & Imprecision & Other considerations & & \\
\hline Symptoms & $6 \mathrm{CSs}$ & 95,169 & Serious $^{1}$ & Serious $^{2}$ & Not serious & Serious $^{3}$ & None & $36 \%(16 \%, 56 \%)$ & $\oplus \bigcirc \bigcirc \bigcirc$ very low \\
\hline Prevention and therapy & $7 \mathrm{CSs}$ & 96,539 & Serious $^{1}$ & Serious $^{2}$ & Not serious & Not serious & None & $23 \%(13 \%, 33 \%)$ & $\oplus \oplus \bigcirc \bigcirc$ low \\
\hline Psychological problems & $4 \mathrm{CSs}$ & 4,158 & Serious $^{1}$ & Serious $^{2}$ & Not serious & Serious $^{3}$ & None & $22 \%(1 \%, 43 \%)$ & $\oplus \bigcirc \bigcirc \bigcirc$ very low \\
\hline Services and advice & $4 \mathrm{CSs}$ & 4,158 & Serious $^{1}$ & Serious $^{2}$ & Not serious & Not serious & None & $16 \%(5 \%, 28 \%)$ & $\oplus \oplus \bigcirc \bigcirc$ low \\
\hline Policy & $2 \mathrm{CSs}$ & 78,683 & Serious $^{1}$ & Serious $^{2}$ & Not serious & Not serious & None & $18 \%(8 \%, 29 \%)$ & $\oplus \oplus \bigcirc \bigcirc$ low \\
\hline Complaint and advice & $5 \mathrm{CSs}$ & 94,385 & Serious $^{1}$ & Serious $^{2}$ & Not serious & Not serious & None & $8 \%(0 \%, 20 \%)$ & $\oplus \oplus \bigcirc \bigcirc$ low \\
\hline $\begin{array}{l}\text { Epidemic situation and } \\
\text { public problem }\end{array}$ & $4 \mathrm{CSs}$ & 93,366 & Serious $^{1}$ & Serious $^{2}$ & Not serious & Not serious & None & $8 \%(6 \%, 10 \%)$ & $\oplus \oplus \bigcirc \bigcirc$ low \\
\hline
\end{tabular}

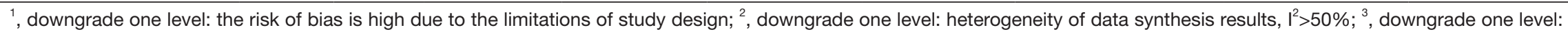
the confidence interval is too wide. $\mathrm{Cl}$, confidence interval; CS, cross-sectional study. 